11

\title{
Условия формирования примесных сверхструктур при фазовых переходах
}

\author{
(c) А.А. Чеврычкина, Н.М. Бессонов, А.Л. Корженевский \\ Институт проблем машиноведения РАН, \\ Санкт-Петербург, Россия \\ ฯ E-mail: alekorzh@mail.ru
}

Поступила в Редакцию 26 февраля 2019 г.

В окончательной редакции 18 марта 2019 г.

Принята к публикации 19 марта 2019 г.

Целью работы является представление условий, необходимых для возникновения слоистых периодических примесных сверхструктур в результате твердотельных фазовых превращений, сопровождающихся изменением концентрации примеси, в общей аналитической форме. В рамках капиллярно-волновой модели получены в явном виде выражения для области значений градиента температуры и средней скорости межфазной границы, внутри которой скорость границы переходит в режим периодических колебаний. Выведена формула для пространственного периода примесной сверхструктуры, появление которой обусловлено этими колебаниями.

Ключевые слова: фазовые переходы, подвижные примеси, капиллярно-волновая модель, примесные сверхструктуры, межфазная граница.

DOI: 10.21883/FTT.2019.10.48268.391

\section{1. Введение}

Совокупность вопросов, связанных с количественными расчетами влияния кинетических факторов на микроструктуру материалов, формирующихся в результате фазовых переходов, составляет одну из наиболее фундаментальных задач физики твердого тела. Ее решение чрезвычайно важно также и для разработки технологических методик, позволяющих надежно контролировать создание новых материалов с заданными микроструктурными параметрами.

Одним из этих кинетических факторов являются подвижные примеси, имеющиеся изначально или целенаправленно введенные в исходный материал. Как хорошо известно, в кристаллах, выращенных из растворов и расплавов, часто видны так называемые „примесные полоски“. Это явление давно наблюдалось во многих материалах совершенно разных классов и типов: полупроводниках [1], диэлектриках [2], минералах [3], коллоидах [4]. Позже, чем в остальных классах, оно было обнаружено и при быстрой направленной солидификации (rapid directional solidification (RDS)) металлических сплавов, образующиеся структуры были названы „полосатыми структурами“ („,banded structures“, BS) [5].

Необходимо отметить, что во многих случаях эти упорядоченные пространственно-неоднородные структуры возникали при постоянных внешних условиях, т.е. происходили процессы самоорганизации. Их наблюдавшееся сходство позволило предположить, что для всех них имеется ограниченный набор механизмов, имеющих универсальный характер. Непосредственную же динамическую причину формирования примесных слоистых сверхструктур установили экспериментально: примесь откладывается неоднородным образом из-за непостоянства скорости движения межфазной границы. Таким образом, необходимо было понять механизм неустойчивости равномерного движения границы и затем количественно рассчитать характеристики ее реальной динамики.

Дальше, чем для других случаев, здесь удалось продвинуться в анализе формирования полосатых структур в металлических сплавах, полученных из их расплавов путем RDS. Кратко отметим его наиболее важные этапы. Экспериментально было установлено, что концентрация примеси в твердом продукте RDS существенно отличается от равновесной (см., например, [6]), при этом ее значение с ростом скорости приближается к равновесному значению в исходном расплаве. Объяснение этого эффекта, получившего название „эффект захвата примеси“ (,solute trapping“), было получено в рамках предельно упрощенной, но по своему характеру микроскопической модели Азиза [7]. Выведенная в [7] формула для зависящего от постоянной скорости границы $V$ коэффициента неравновесного распределения примеси $k(V)$ была использована Бетингером и др. [8] для расчета коэффициента наклона так называемой „кинетической линии ликвидуса“ $m_{L}(k)$, что позволило определить температуру на движущейся границе. Формула для коэффициента наклона кинетического ликвидуса $m_{L}(k)$ в [8] была получена как в предположении об отсутствии диссипативных процессов внутри слоя межфазной границы (так называемого „примесного торможения“, что является в действительности очень жестким ограничением), так и при их наличии (то есть, с учетом примесного торможения). Первый предельный случай получил название „without solute drag“, а второй — „with 
solute drag“. Используя обе функции $k(V)$ и $m_{L}(k)$ в качестве неравновесных граничных условий на фронте расплав-кристалл в задаче Стефана, можно провести анализ линейной устойчивости плоского фронта, движущегося со средней заданной скоростью $V_{p}$ в поле постоянного градиента температуры $G$. Такой анализ был проделан в работе [9], в которой, однако, „функции отклика“ $k(V)$ и $m_{L}(k)$ рассматривались как независимые друг от друга феноменологические выражения. В работе [10] было показано, что для моделей типа [7] (так называемая модель непрерывного роста (continuous growth model, CGM)) определенная диссипация энергии, как правило, существует и приводит к появлению вязкой силы трения, тормозящей движение границы.

Несмотря на особое место, занимаемое ею в ряду моделей, по-разному оценивающих вклад диссипативных процессов, ведущих к появлению примесного торможения, для конкретизации вида функции $m_{L}$ при анализе линейной устойчивости в работе [11] была использована модель „without solute drag“. Выведенное для нее в неявной форме дисперсионное уравнение было решено численно. Решение показало, что помимо хорошо известной морфологической неустойчивости Маллинза и Секерки, существующей и в предположении локального термодинамического равновесия на границе [12], учет неравновесного эффекта захвата примеси приводит к появлению дополнительной неустойчивости, возникающей в результате бифуркации Хопфа. При этом максимальной скорости развития неустойчивости отвечали осцилляции скорости границы как целого, что указывало на возможность формирования в продукте реакции позади нее системы параллельных границе слоев, с периодически меняющимся распределением концентрации примеси (названных solute bands). Учитывая ограниченность варианта модели выбранного в [11] (игнорирование эффекта примесного торможения и использование результатов CGM, применимых лишь для движения границы с постоянной скоростью), Конти провел численные расчеты для более общей модели фазового поля [13], которая была детально разработана для количественного описания двух основных неравновесных эффектов захвата примеси и примесного торможения в бинарных сплавах в работе [14]. Его расчеты показали, что в такой модели, вообще не использующей каких-либо определяющих уравнений на размытой межфазной границе, при некоторых выбранных значениях $V_{p}$ и $G$ действительно возникают слоистые периодические примесные структуры, но вместе с тем их параметры заметно отличались от предсказанных в модели [11]. Однако, поскольку использованная Конти модель фазового поля содержала большое количество параметров (это общее свойство любых фазовых моделей, функционалы которых всегда необходимо параметризовать для проведения вычислений), было затруднительно сформулировать условия на область их значений, при выполнении которых появляются такие структуры, без проведения численных расчетов для огромного массива исходных данных.
При солидификации сплавов область высоких скоростей вблизи границы абсолютной стабильности, где post-mortem наблюдались BS, долгое время оставалась недоступной для прямых (in situ) экспериментальных исследований. Лишь в последние годы была разработана методика динамической просвечивающей электронной микроскопии (dynamic transmission electron microscopy, DTEM) с достаточно высоким пространственным и временным разрешением, позволяющая проведение таких экспериментов [15-17]. Например, с ее помощью in situ впервые удалось проследить, как осцилляционная неустойчивость плоской границы инициирует возникновение и временную эволюцию BS в сплаве $\mathrm{Al}-\mathrm{Cu}$, а также измерить значения мгновенных скоростей межфазной границы.

Кроме того, сравнительно недавно было осознано, что изучение неравновесных эффектов захвата примеси и примесного торможения в области скоростей вблизи границы абсолютной стабильности гораздо легче проводить при диффузионных переходах между твердыми фазами, поскольку из-за медленности процесса диффузии в них значения характерных скоростей падают на несколько порядков [18]. Например, в работе [19] было установлено, что в сплаве $\mathrm{Fe}-\mathrm{Co}$ межфазная поверхность $\gamma / \delta$ при достижении скорости, отвечающей границе абсолютной стабильности, испытывает морфологический переход от ячеистого к плоскому фронту. При этом было отмечено, что, хотя при выбранном значении концентрации кобальта и градиента температуры условия возникновения осцилляционной неустойчивости не были выполнены и появления BS не наблюдалось, но в принципе область неустойчивости в подобных экспериментах вполне достижима.

Вместе с тем, в связи с необходимостью разработки надежных способов контроля создания технологически важных неравновесных микроструктур, в настоящее время проводятся эксперименты и по быстрой солидификации интерметаллических сплавов [20].

В свою очередь, недавно в модельных теоретических работах [21-23] впервые были аналитически рассчитаны пространственные профили концентрации в примесных полосах и динамика BS как при постоянной, так и при осциллирующей скорости протяжки образца в поле заданного температурного градиента.

Совокупность перечисленных выше недавних результатов является стимулом для создания более последовательного количественного описания как самой области, где возможно формирование диффузионных BS, так и играющих при высоких скоростях ключевую роль неравновесных эффектов захвата примеси и примесного торможения в рамках все более реалистичных моделей, свободных от ряда прежних феноменологических предположений.

В настоящей работе мы проведем анализ линейной устойчивости движения плоской границы конечной толщины, разделяющей фазы с разной концентрацией примеси, которая находится в поле градиента темпера- 
туры $G$, движущегося с заданной скоростью $V_{p}$. Мы выведем простые аналитические формулы, которые хорошо аппроксимируют формы областей неустойчивости границы, а также найдем значения пространственных периодов примесных слоистых сверхструктур $R$ при любых заданных значениях управляющих параметров $V_{p}$ и $G$ и различных равновесных профилях концентрации примеси в окрестности границы.

\section{2. Уравнения движения межфазных границ в моделях фазового поля и модели капиллярных волн}

Стандартный набор моделей фазового поля (МФП), предназначенный для описания динамики межфазных границ в материалах с подвижными примесями, задается уравнениями движения градиентного типа:

$$
\begin{gathered}
H=\int d^{3} r\left\{\frac{\sigma}{\xi}\left[\frac{\xi^{2}}{2}(\nabla \varphi)^{2}+W(\varphi)\right]\right. \\
\left.+\frac{\kappa(T)}{2}[C-U(\varphi, T)]^{2}\right\}, \\
\partial_{t} \varphi=-\Gamma \frac{\delta H}{\delta \phi}, \\
\partial_{t} C=\nabla\left(\frac{D}{k}\right) \nabla \frac{\delta H}{\delta C},
\end{gathered}
$$

где $\Gamma-$ кинетический коэффициент, $D-$ коэффициент диффузии примеси, эффективный гамильтониан $H$ содержит „двухъямную“ функцию фазового поля $W(\varphi)$ и „одноямную“ $U(\varphi, T)$. Первая из них позволяет описать сосуществование двух разных фаз, а вторая - учесть наличие в них разных уровней концентрации примеси $C$. Коэффициенты $\sigma$ и $\xi$ задают поверхностное натяжение и ширину переходной межфазной области. В случае разбавленного бинарного сплава функция $\kappa(T)$ имеет вид:

$$
\kappa(T)=-\left(\frac{\partial C}{\partial T}\right)^{-1} \frac{L}{T_{c}} \frac{1}{\Delta C(T)}
$$

где $L, T_{c}$ и $\Delta C(T)$ обозначают теплоту превращения, температуру перехода и концентрационную ширину области гистерезиса соответственно.

Для получения аналитических результатов гораздо удобнее, однако, работать с так называемой капиллярно-волновой моделью (КВМ), определенной уравнениями

$$
\begin{aligned}
H=\frac{\sigma}{2} \int d^{2} x(\partial Z)^{2} & +\frac{\kappa(T)}{2} \int d^{3} r\left[C-C_{e}(z-Z)\right]^{2}, \\
\partial_{t} Z & =\Lambda\left(F-\frac{\delta H}{\delta Z}\right), \\
\partial_{t} C & =\nabla\left(\frac{D}{k}\right) \nabla \frac{\delta H}{\delta C},
\end{aligned}
$$

где $Z(x)$ - координата границы, кинетический коэффициент $\Lambda=\Gamma \xi^{2} / \sigma$, функция $C_{e}$ описывает равновесное распределение примеси в присутствии границы. Движущая сила $F$, действующая на границу, определяется как $F=L\left(T_{c}-T\right) / T_{c}$. Значение локальной температуры $T$ определяется заданной средней скоростью границы $V_{p}$ и фиксированным градиентом температуры $G$

$$
T=T_{c}+G\left(z-V_{p} t\right) .
$$

В работе [24] был приведен детальный математический вывод КВМ (3) из модели фазового поля (1) при выполнении условий малости значений безразмерных параметров $1 / p \ll 1, \gamma \ll 1$, определенных ниже. Физический смысл этих условий таков: первое означает, что максимальная скорость межфазной границы в отсутствие примеси много больше диффузионной: $V_{c}=\Lambda \xi \gg V_{d}=D / \xi$ (оно выполняется для границ с высокой подвижностью, в частности, для границ в металлических сплавах [25], где $V_{c} \sim 10^{3} \mathrm{~m} / \mathrm{s}$, a $V_{d} \sim 1-10 \mathrm{~m} / \mathrm{s}$ ), а второе означает, что температурный интервал сосуществования фаз $\Delta T \ll T_{c}$ (что реализуется в большинстве разбавленных сплавов с номинальной концентрацией $\left.C_{0} \ll 1\right)$.

Удобно ввести безразмерные переменные

$$
\frac{r}{\xi} \rightarrow r, \quad \frac{D t}{\xi^{2}} \rightarrow t, \quad \frac{2\left(C-C_{0}\right)}{\Delta C} \rightarrow C,
$$

и независимые безразмерные параметры:

$$
\gamma=\left(\frac{\Delta C}{2}\right)^{2} \frac{\kappa \xi}{\sigma}, \quad p=\frac{V_{c}}{V_{d}}, \quad g=\frac{\xi^{2} L}{\sigma T_{c}} G .
$$

При этом безразмерная скорость $v$ будет измеряться в единицах диффузионной скорости $V_{d}: v=V / V_{d}$. Во введенных безразмерных переменных уравнения движения границы приобретают вид

$$
\begin{gathered}
\frac{1}{p} \partial_{t} Z=F(Z)-\gamma \int d z C_{e}^{\prime}(z-Z)\left[C-C_{e}(z-Z)\right], \\
\partial_{t} C=D \nabla^{2}\left[C-C_{e}(z-Z)\right] .
\end{gathered}
$$

Отметим, что здесь хорошо видны преимущества анализа динамики межфазных границ в рамках КВМ по сравнению с МФП. В КВМ эта задача четко распадается на две: сначала в условиях термодинамического равновесия вычисляются или измеряются профиль сегрегации примеси, величина поверхностного натяжения, ширина границы и коэффициент диффузии. Затем эти данные используются как вводные для решения уравнения движения границы в конкретном материале. Важно, что в отличие от гибкой, но излишне обобщенной схемы МФП, а также феноменологических моделей типа [7-10], в КВМ не возникает никакой неоднозначности при вычислении эффективных сил трения, действующих на границу (по традиции их принято называть „кинетическим переохлаждением“'). Их расчет сводится к решению диффузионного уравнения (8) и подстановке этого решения в последний член в уравнении (7). После этого становится очевидным, что уравнение (7) является не чем иным, как естественным условием баланса всех сил, приложенных к границе в неравновесных условиях. 


\section{3. Анализ линейной устойчивости равномерного движения плоской межфазной границы}

Если предположить, что граница движется с той же постоянной скоростью, что и изотермы теплового поля $V_{p}$, то уравнение силового баланса (7) принимает вид алгебраического равенства:

$$
\begin{gathered}
\frac{1}{p} v_{p}=F\left(v_{p}\right)+f\left(v_{p}\right)-f(0), \\
f(v)=-\gamma \int d \xi C_{e}^{\prime}(\xi) C(\xi, v), \\
F(v)=F\left(v_{p}\right)-g\left(Z-v_{p} t\right),
\end{gathered}
$$

где координата $\xi=z-Z$.

Устойчивость его решения $Z=Z_{0}+v_{p} t$ относительно малых отклонений профилей границы $h$ и концентрации примеси $c$ в форме

$$
\begin{gathered}
h(\mathbf{x}, t)=\tilde{h}(\mathbf{q}, \omega) \exp (i \mathbf{q} \mathbf{x}+\omega t), \\
c(\xi, \mathbf{q}, \omega)=\tilde{c}(\xi, \mathbf{q}, \omega) \exp (i \mathbf{q} \mathbf{x}+\omega t)
\end{gathered}
$$

определяется знаком $\operatorname{Re} \omega$. Уравнения для амплитуд $\tilde{h}(\mathbf{q}, \omega)$ и $\tilde{c}(\xi, \mathbf{q}, \omega)$ имеют вид:

$$
\begin{aligned}
& \left(\omega+p q^{2}+g\right) \tilde{h}(\mathbf{q}, \omega)=-p \gamma \int d \xi C_{e}^{\prime}(\xi) \tilde{c}(\xi, \mathbf{q}, \omega) \\
& \left(\omega+q^{2}-v \partial \xi-\partial_{\xi}^{2}\right) \tilde{c}(\xi, \mathbf{q}, \omega) \\
& \quad=C^{\prime \prime}(\xi)\left(\omega+q^{2}\right) \tilde{h}(\mathbf{q}, \omega)-C_{e}^{\prime}(\xi) q^{2} \tilde{h}(\mathbf{q}, \omega) .
\end{aligned}
$$

Приравнивая нулю детерминант линейных уравнений на эти амплитуды, получаем дисперсионное уравнение на $\omega(\mathbf{q})$ :

$$
\begin{aligned}
\frac{\omega}{p}+q^{2}+g & -v[f(v+\lambda)-f(v)] \\
= & \frac{\lambda^{2}-q^{2}}{v+2 \lambda}[f(v+\lambda)+f(\lambda)],
\end{aligned}
$$

содержащее ту же самую функцию $f$, которая входит в алгебраический баланс сил (9), но при значениях аргументов, зависящих от функции $\lambda(v)$ :

$$
\lambda=-\frac{v}{2}+\sqrt{\frac{v^{2}}{4}+\omega+q^{2}} .
$$

Поскольку $\omega$ входит в дисперсионное уравнение (14) неявным образом, то создается впечатление, что получить решение для $\omega$ можно только после того, как из уравнения (10) будет явным образом найдена функция $f(v)$ при фиксированной форме профиля равновесной концентрации $C_{e}(z-Z)$. Иными словами, кажется, что получить выражение для $\operatorname{Re} \omega$ в общем виде невозможно, и контур области неустойчивости, внутри которой возникает слоистая примесная сверхструктура, потребует для каждого изучаемого примесного материала отдельного рассмотрения, причем скорее всего, выполнимого лишь посредством весьма обременительных численных расчетов.

Тем не менее, ниже мы покажем, что с хорошей точностью можно найти область появления примесных сверхструктур в универсальной аналитической форме, причем зависимость их пространственных периодов от скорости изотерм $V_{p}$ и градиента температуры $G$ на основной части контура области имеет очень простой вид.

Поиск решений уравнения (14) начнем, однако, с более простого случая, когда при условии $\operatorname{Re} \omega=0$ также и $\operatorname{Im} \omega=0$. Такая ситуация, в частности, имела место в расчете Маллинза и Секерки (МC) [12] при анализе устойчивости бесконечно тонкой границы, находящейся в состоянии локального термодинамического равновесия. Учтем, что обнаруженная ими неустойчивость исчезала при скоростях выше $V_{a}=D / d_{0}$, где в наших обозначениях „химическая“ длина $d_{0}=\xi / \gamma$. Используя определение (6), получаем оценку для $\gamma \propto \Delta T / T_{c} \ll 1$. Отсюда следует, что определяющая нижнюю границу так называемой „области абсолютной устойчивости“ скорость $V_{a} \ll V_{d}=D / \xi$, следовательно, для неустойчивости типа МС, значения безразмерной скорости $v \ll 1$. При таких скоростях длина волны МС велика, так что ассоциированный с ней безразмерный волновой вектор $q \ll 1$. Предположим, что и для границ с конечной толщиной неравенства $v \ll 1$ и $q \ll 1$ выполняются (что это действительно так, будет видно a posteriori). Используя условие $d \omega / d q=0$, определяющее величину $q$ для наиболее быстро растущей моды возмущения, положим в уравнении (14) также $\omega=0$ и перепишем его в виде:

$$
\begin{aligned}
g+\lambda^{2}+\lambda v & -v[f(v+\lambda)-f(v)] \\
& =\frac{-\lambda v}{v+2 \lambda}[f(v+\lambda)+f(\lambda)] .
\end{aligned}
$$

Сохранив в разложениях Тейлора для функций $g(v+\lambda), g(\lambda), g(v)$ в окрестности нуля члены вплоть до третьего порядка включительно, получаем после выполнения довольно длинных алгебраических выкладок, которые мы опускаем, выражение для функции $\lambda(v)$ :

$$
\lambda(v)=\sqrt[3]{-f(0) v^{2} / 4}-\frac{v}{2} .
$$

Так как оно зависит не от вида всей функции $f(v)$, а только от ее термодинамического значения $f(0)=-2 \gamma$, то ясно, что после подстановки выражения (17) в (16) контур области неустойчивости МC $g=g(v)$ определится универсальным образом. В частности, ее максимальная „высота“ $v_{a}$, отвечающая условию восстановления устойчивости плоской границы $q=\sqrt{\lambda(\lambda+v)}=0$, определится как $v_{a}=4 \gamma$.

Поскольку параметр $\gamma \ll 1$, то наше предположение о возможности разложения функций $f$ в уравнени- 


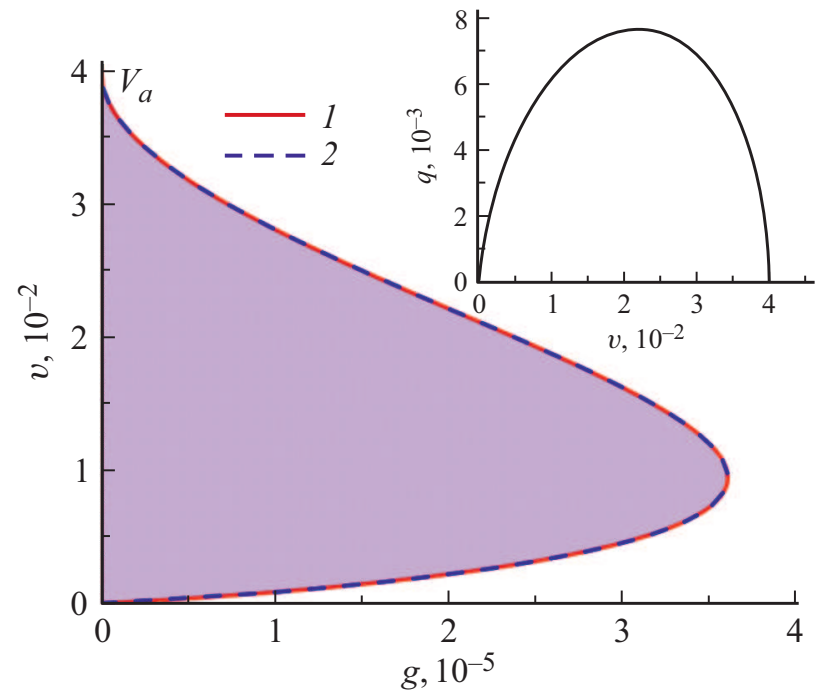

Рис. 1. Контур области МС-неустойчивости: 1 - численное решение точного дисперсионного уравнения (14), 2 - его аппроксимация функцией (18). Значения параметров: $p=100$, $\gamma=0.01$. На вставке - зависимость волнового вектора МС от безразмерной скорости.

ях (16) становится оправданным. Соответственно, область неустойчивости МС описывается кривой

$$
g=-\lambda\left[\lambda+v-\frac{4 v \lambda}{v+2 \lambda}\right]
$$

Тем самым мы показали, что этот результат для области классической неустойчивости Маллинза и Секерки, полученной ими в предположении локального термодинамического равновесия на „резкой“ границе (т.е. в наших обозначениях для модели с $\xi=0$, $f(v)=f(0)=-2 \lambda)$, не зависит от деталей профиля равновесного распределения примеси $C_{e}(z-Z)$ в окрестности границы и остается справедливым и при учете неравновесных эффектов (с относительной точностью $\sim O(\gamma) \ll 1)$. На рис. 1 приведены графики области $\mathrm{MC}$ неустойчивости для функции $f(v)$, имеющей вид

$$
f(v)=-\gamma \frac{(v+2)}{(v+1)^{2}},
$$

который отвечает выбору равновесного примесного профиля:

$$
C_{e}(\zeta)=\Delta C\{\theta(-\zeta)[-1+\exp (\zeta)]+\theta(\xi)[1-\exp (-\zeta)]\}
$$

где $\theta-$ функция Хэвисайда.

На вставке на рис. 1 построена зависимость волнового вектора МC $\mathbf{q}=q(v)$. Видно, что при приближении к границе ,абсолютной устойчивости“ $v \rightarrow v_{a}$ имеем $q \rightarrow 0$, так что для модели с нулевой шириной границы ее плоская форма снова становится устойчивой при $v>v_{a}$.
Однако кроме неустойчивости $\mathrm{MC}$ с $\operatorname{Im} \omega=0$, дисперсионное уравнение (14) обладает еще одной неустойчивой веткой $\omega(\mathbf{q})$, у которой $\operatorname{Im} \omega \equiv \Omega$ не равна нулю. В этом случае комплексное уравнение (14) распадается на два уравнения для его действительной и мнимой части. Поскольку, как мы увидим, интервал скоростей у области неустойчивости для этой ветки простирается до значений безразмерной скорости $v \sim 1$, то разложение, использованное выше для определения формы области МС-неустойчивости, становится неэффективным. Однако оказывается, что мнимая часть $\omega(\mathbf{q})$ является достаточно малой, так что можно вести разложение по параметру $\epsilon \equiv 2 i \Omega / v^{2}$. Ограничиваясь в разложении для $\lambda$ кубическим членом по $\epsilon$, получаем, опуская промежуточные алгебраические выкладки, выражения

$$
\begin{gathered}
g=\left(\frac{1}{p}-f^{\prime}(v)\right) \frac{v^{2} B(v)}{4 A(v)}, \\
\Omega^{2}=\left(f^{\prime}(v)-\frac{1}{p}\right) \frac{v^{5}}{4 A(v)},
\end{gathered}
$$

где функции $A$ и $B$ определены как

$$
\begin{aligned}
A(v)= & -f(v)-f(0) \\
& +\frac{v}{4}\left(3 f^{\prime}(v)+f^{\prime}(0)-v f^{\prime \prime}(0)+\frac{v^{2}}{6} f^{\prime \prime \prime}(v)\right), \\
B(v) & =f(v)+f(0)-v_{f^{\prime}(v)}+\frac{v^{2}}{2} f^{\prime \prime}(v)
\end{aligned}
$$

где штрихом обозначена производная по аргументу.

Из формулы (22) мы видим, что на контуре $g(v)$ можно выделить две части, где $\Omega$ асимптотически стремится к нулю: во-первых, это область низких скоростей $v \rightarrow 0$, во-вторых, область малых градиентов температуры $g \rightarrow 0$, когда скобка $\left(1 / p-f^{\prime}(v)\right) \rightarrow 0$ вблизи максимального значения скорости $v=v_{m}$ на контуре. В этих областях формулы (21), (22) приобретают очень простой вид. При $v \rightarrow 0$ имеем

$$
\begin{aligned}
& \left(g=\left(f^{\prime}(0)-\frac{1}{p}\right) \frac{v^{2}}{4},\right. \\
& \Omega^{2}=v^{5} \frac{\left(f^{\prime}(0)-\frac{1}{p}\right)}{16 \gamma},
\end{aligned}
$$

то есть асимптотически $g \sim v^{2}, \Omega \sim g^{5 / 4}$, а при $v \rightarrow v_{m}$ из (21) получаем асимптотику $\Omega \sim g^{1 / 2}$.

Из условия $d g / d v=0$ следует, что максимально возможное значение градиента температуры на контуре достигается при значении скорости $v^{*}<v_{m}$, которое не пропорционально какому-либо малому параметру, т.е. $v_{m} \sim O(1)$. Из уравнения (21) получаем, что величина $g\left(v^{*}\right) \sim \gamma \ll 1$. Поскольку обе степенные асимптотики для $\Omega$ должны гладко сшиваться при этом малом $g=g\left(v^{*}\right)$, то и максимальное значение $\Omega$ при $v \approx v^{*}$ 
мало в меру малости $\gamma$. Это обстоятельство оправдывает применение разложения по $\varepsilon$ в уравнении (14) и подразумевает, что полученные с его помощью выражения для контура области, внутри которой возникают осцилляции скорости межфазной границы $V$ вокруг заданного значения $V_{p}(21)$, а также для частоты этих осцилляций $\Omega(22)$ обладают достаточно хорошей точностью. На рис. 2 проведено сравнение формы области осцилляционной неустойчивости (21) с результатами численного решения для точного дисперсионного уравнения (14), в котором функция $f(v)$ была выбрана в виде (19). На рис. 3 проведено такое же сравнение для частот колебаний, а из рис. 4 видно, что их значения хорошо описываются степенными асимптотиками (24)

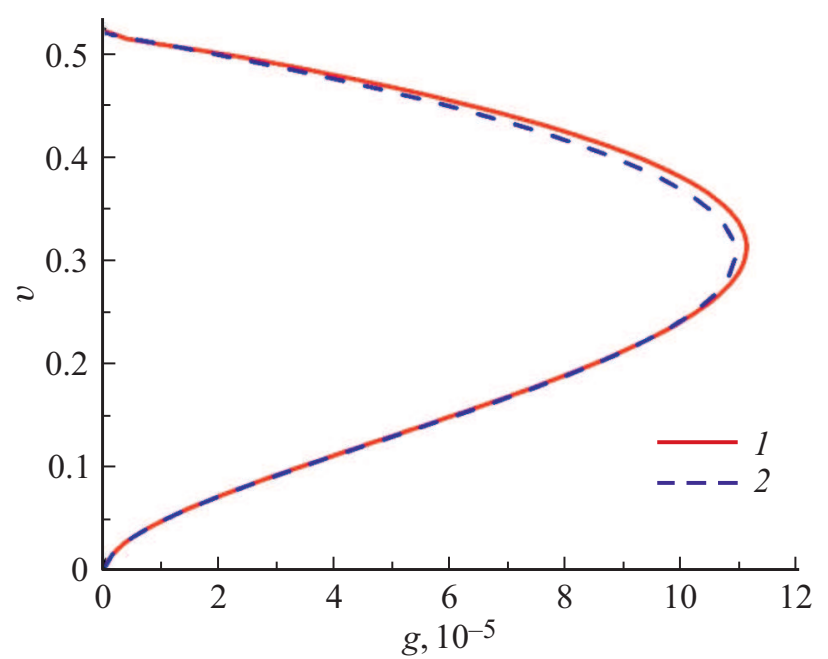

Рис. 2. Контур области осцилляционной неустойчивости: 1 численное решение точного дисперсионного уравнения (14), 2 - его аппроксимация формулой (21).

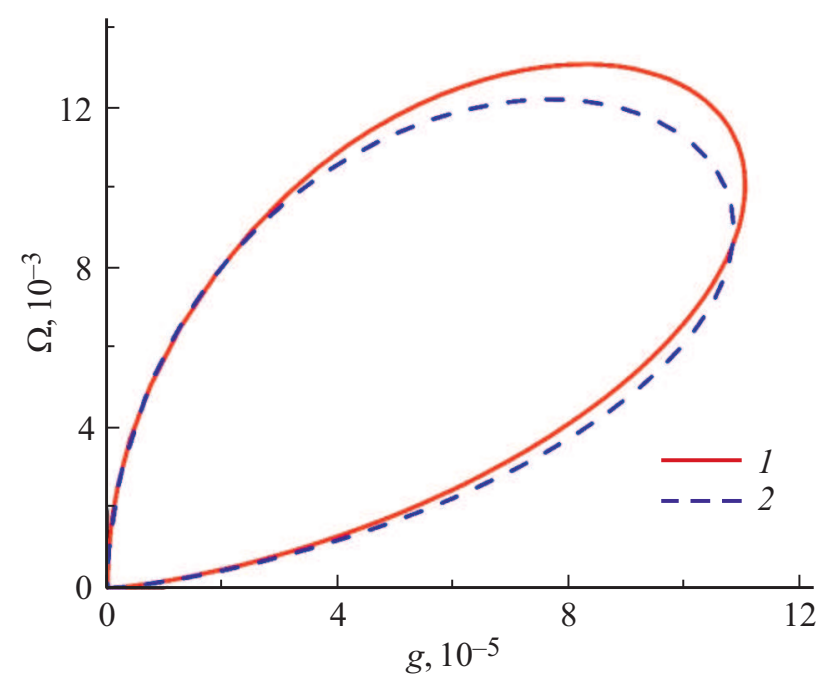

Рис. 3. Зависимость частоты $\Omega$ от градиента температуры $g$ для осцилляционной неустойчивости: 1 - ее численный расчет из точного дисперсионного уравнения (14), 2 - по формуле (22).

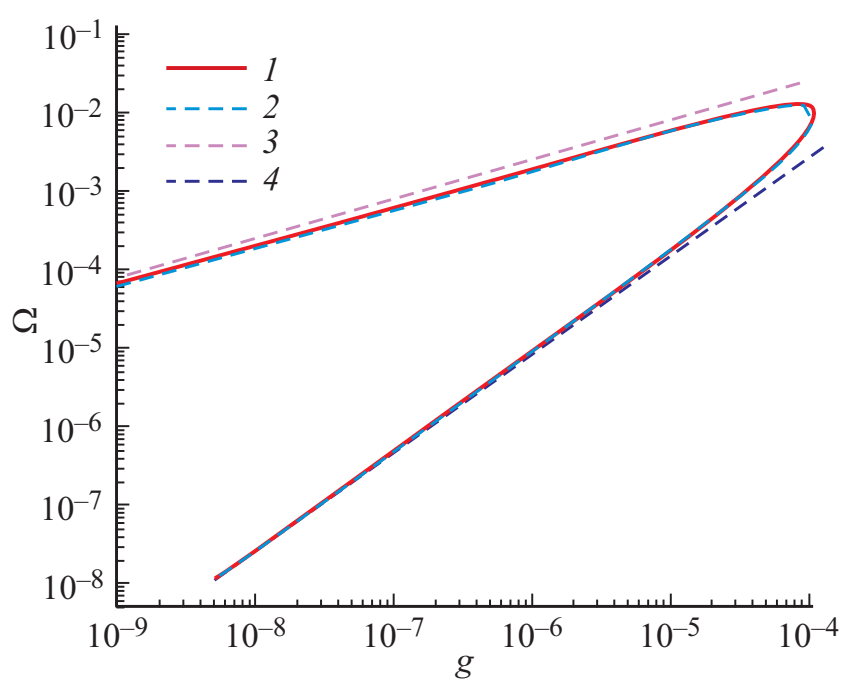

Рис. 4. Зависимость частоты $\Omega$ для осцилляционной неустойчивости от градиента температуры $g$ в двойных логарифмических координатах: 1 - численное решение точного дисперсионного уравнения (14), 2 - по формуле (22), 3, 4 соответствуют степенным асимптотикам (22) и (24).

для значений параметров на большей части контура области осцилляционной неустойчивости.

Таким образом, графики на рис. 1-4 подтверждают, что простые аналитические формулы (21)-(24) хорошо аппроксимируют формы обеих областей неустойчивости, а также величины пространственных периодов примесных слоистых сверхструктур, определяемых формулой $R=2 \pi V / \omega$, при любых заданных значениях управляющих параметров $V_{p}$ и $G$.

\section{4. Заключение}

В рамках капиллярно-волновой модели выведены аналитические выражения для двух областей неустойчивости плоской „размытой“ межфазной границы разбавленного сплава, движущейся с заданной средней скоростью в поле постоянного градиента температуры. Показано, что форма области неустойчивости Маллинза и Секерки не зависит от деталей профиля равновесного распределения примеси вблизи границы с точностью до малых поправок. Еще одна „внутренняя“ диффузионная неустойчивость возникает только при учете конечной ширины межфазной границы, обладающей при фазовом переходе в чистом кристалле высокой подвижностью. Аналитически определены границы области, отвечающей этой неустойчивости, существующей в гораздо более широких интервалах скоростей и градиентов температуры, чем неустойчивость Маллинза и Секерки. Возникновение этой неустойчивости сопровождается появлением периодических осцилляций скорости границы относительно ее среднего заданного значения и формированием регулярной слоистой примесной сверхструктуры. Получена аналитическая формула, определя- 
ющая пространственный период этой сверхструктуры на контуре области ее существования. Полученные в этой статье результаты являются необходимым шагом для создания нелинейной теории, позволяющей количественно рассчитывать профили примесных сверхструктур в глубине области неустойчивости и целенаправленно управлять их свойствами. Аналитические и численные результаты, полученные нами для нелинейного режима, будут приведены в следующей статье.

\section{Финансирование работы}

Работа выполнена при финансовой поддержке РФФИ (грант № 17-02-00365).

\section{Конфликт интересов}

Авторы заявляют, что у них нет конфликта интересов.

\section{Список литературы}

[1] G. Dhanaraj, K. Byrappa, V. Prasad, M. Dudley. Springer Handbook of Crystal Growth, Springer-Verlag Berlin Heidelberg. (2010). 1818 p.

[2] H.J. Scheel, P. Capper. Crystal Growth Technology. WILEYVCH Verlag GmbH \& Co. KGaA, Weinheim (2008). 509 p.

[3] M. Shore, A.D. Fowler. Can. Mineral. 34, 1111 (1996).

[4] J.A.W. Elliot, S.S.L. Peppin. Phys. Rev. Lett. 107, 168301 (2011).

[5] W.J. Boettinger, D. Schechtman, R.J. Schaffer, F.S. Biancaniello. Metal. Trans. A 15, 55 (1984).

[6] M.J. Aziz, J.Y. Tsao, M.O. Thomson, P.S. Peercy, C.W. White. Phys. Rev. Lett. 56, 2489 (1986).

[7] M.J. Aziz. J. Appl. Phys. 53, 1158 (1982).

[8] M.J. Aziz, W.J. Boettinger. Acta Met. Mater. 42, 527 (1994).

[9] S.R. Coriell, R.F. Sekerka. J. Cryst. Growth 61, 499 (1983).

[10] M.J. Aziz, T. Kaplan. Acta Met. 36, 2335 (1988).

[11] G.J. Merchant, S. Davis. Acta Met. Mater. 38, 2683 (1990).

[12] W.W. Mullins, R.F. Sekerka. J. Appl. Phys. 35, 444 (1964).

[13] M. Conti. Phys. Rev. E 56, R6267 (1997); 58, 2071 (1998).

[14] N.A. Ahmad, A.A. Wheeler, W.J. Boettinger, McFadden. Phys. Rev. E 58, 3436 (1998).

[15] J.T. McKeown, A.K. Kulovits, C. Liu, K. Zweiacker, B.W. Reed, T. LaGrange, J.M.K. Wiezorek, G.H. Campbell. Acta Mater 65, 56 (2014).

[16] J.T. McKeown, K. Zweiacker, C. Liu, D.R. Coughlin, A.J. Clarke, J.K. Baldwin, J.W. Gibbs, J.D. Roehling, S.D. Imhoff, P.J. Gibbs, D. Tourret, J.M.K. Wiezorek, G.H. Campbell. JOM 68, 3, 985 (2016).

[17] K.W. Zweiacker, C. Liu, M.A. Gordillo, J.T. McKeown, G.H. Campbell, J.M.K. Wiezorek. Acta Mater. 145, 71 (2018).

[18] M. Asta, C. Beckermann, A. Karma, W. Kurz, R. Napolitano, M. Plapp, G. Purdy, M. Rappaz, R. Trivedi. Acta Mater. 57, 941 (2009).

[19] A. Jacot, M. Sumida, W. Kurz. Acta Mater. 59, 1716 (2011).
[20] J. Jang, H. Wang, W. Kuang, Y. Zhang, S. Li, Y. Zhao, D.M. Herlach. Acta Mater. 148, 86 (2018).

[21] A.L. Korzhenevskii, R. Bausch, R. Schmitz. Phys. Rev. Lett. 108, 046101 (2012).

[22] A.L. Korzhenevskii, R. Bausch, R. Schmitz. Phys. Rev. E 85, 021605 (2012).

[23] R.E. Rozas, A.L. Korzhenevskii, J. Horbach. J. Phys. Condens. Matter 28, 035001 (2016).

[24] A.L. Korzhenevskii, R. Bausch, R. Schmitz. Phys. Rev. E 83, 041609 (2011).

[25] J.Q. Broughton, G.H. Gilmer, K.A. Jackson. Phys. Rev. Lett. 49, 1496 (1982).

Редактор Е.Ю. Флегонтова 